Notre Dame Journal of Formal Logic

Volume 41, Number 1, 2000

\title{
ESSENTIAL FORCING GENERICS
}

\author{
STEPHANIE CAWTHORNE AND DAVID KUEKER
}

\begin{abstract}
We use model theoretic forcing to study and generalize the construction of $(K, \leq)$-generic models introduced by Kueker and Laskowski. We characterize the $(K, \leq)$-generic models in terms of forcing and introduce a more general class of models, called essential forcing generics, which have many of the same properties.
\end{abstract}

\section{Introduction}

$(\mathrm{K}, \leq)$-generic models were introduced in Kueker and Laskowski [ 4 ] as a generalization of the classical Fraisse construction of homogeneous-universal models. In this paper we use a form of model-theoretic forcing to study and generalize this construction. In particular, we characterize the $(K, \leq)$-generic models in terms of forcing and introduce a more general class of models, the essential forcing generics, which have many of the same properties. This paper is intended to demonstrate that in addition to being a useful tool for studying $(K, \leq)$-generics, forcing leads to generalizations of interest.

In Section 2 we introduce the sort of forcing we use throughout the paper (a generalization of Robinson's finite forcing [5]) and state the basic facts on the existence and the properties of forcing generics.

In Section 3 we define the essential forcing generics as countable models which are forcing generic with respect to $L_{\omega_{1} \omega}$. We characterize them as "homogeneousuniversal" for a weak form of homogeneity (Theorem 3.5) and identify the ( $K, \leq)$ generics as a specific sort of essential forcing generic (Theorem 3.6).

In Section 4 we study properties of essential forcing generics, obtaining generalizations of many of the results on $(K, \leq)$-generics established in [4].

The results in this paper form part of the first author's doctoral dissertation, Cawthorne [2].

Received July 27, 1999; revised May 27, 2000; printed July 15, 2002

2001 Mathematics Subject Classification: Primary, 03C25; Secondary, 03C50

Keywords: forcing, generic model

(c) 1999 University of Notre Dame 


\section{Forcing with Directed Families of Finite Structures}

Throughout this paper we assume that $L$ is a countable relational language. The assumption that $L$ is relational is not essential but it simplifies the presentation. In [4] the notion of a smooth class $(K, \leq)$ of finite structures, which is used to construct $(K, \leq)$-generic structures, is introduced. We work with directed families, smooth classes which are countable up to isomorphism and satisfy joint embedding.

Definition 2.1 Let $K$ be a class of finite $L$-structures closed under isomorphism and let $\leq$ be a binary relation on $K$. Then $(K, \leq)$ is a directed family of finite structures provided the following hold:

1. $K$ is countable up to isomorphism;

2. $\leq$ is transitive and for all $\mathfrak{B}, \mathfrak{C} \in K, \mathfrak{B} \leq \mathfrak{C}$ implies $\mathfrak{B} \subseteq \mathfrak{C}$;

3. $(K, \leq)$ satisfies joint embedding, that is, for every $\mathfrak{B}_{1}, \mathfrak{B}_{2} \in K$ there are $\mathfrak{E} \in K$ and isomorphic embeddings $f_{i}: \mathfrak{B}_{i} \rightarrow \mathfrak{E}$ so that $f_{i}\left(\mathfrak{B}_{i}\right) \leq \mathfrak{E}$ for $i=1,2$;

4. for each $\mathfrak{B} \in K$ there is a set $p^{\mathfrak{B}}(\bar{x})$ of universal formulas with $|\bar{x}|=|B|$ such that for any $\mathfrak{B} \in K$ with $\mathfrak{B} \subseteq \mathfrak{C}, \mathfrak{B} \leq \mathfrak{C}$ if and only if $\mathfrak{S} \models \varphi(\bar{b})$ for all $\varphi \in p^{\mathfrak{B}}$, where $\bar{b}$ enumerates $B$; we also require $p^{\mathfrak{B}}=p^{\mathfrak{E}}$ whenever $\mathfrak{B} \cong \mathfrak{E}$.

We refer to $\leq$ as strong substructure. Further, we may assume $p^{\mathfrak{A}}(\bar{x})$ contains the basic open diagram of $\mathfrak{A}$, so that if $\mathfrak{B} \models \wedge p^{\mathfrak{A}}\left(\bar{a}^{\prime}\right)$ then defining $f\left(a_{i}\right)=a_{i}^{\prime}$ for all $i<|A|$ yields an isomorphism of $\mathfrak{U}$ onto $\mathfrak{U}^{\prime} \leq \mathfrak{B}$.

By convention, $\mathfrak{A}, \mathfrak{B}, \mathfrak{\complement}$ will always refer to structures in $K$ while $\mathfrak{M}, \mathfrak{N}$ will be used for arbitrary (usually infinite) structures. The universe of $\mathfrak{A}, \mathfrak{M} i$ will be denoted by $A, M$ respectively.

We use directed families $(K, \leq)$ to define a forcing relation where structures in $K$ are the forcing conditions and $\leq$ is the extension relation. The resulting notion of forcing is a straightforward generalization of Robinson's finite forcing (see [5]) and is covered by Keisler's very general treatment (see [3]). In this section we give the basic definitions and facts concerning forcing and forcing generics, specialized to our context. These are almost all standard and the reader is referred to [3] and [5] for more detail.

Definition 2.2 Let $(K, \leq)$ be a directed family of finite structures. We define the relation $\mathfrak{A}$ forces $\theta$, $\mathfrak{U} \Vdash \theta$, for $\mathfrak{A} \in K$ and $\theta \in S n_{L(A)}$, as follows:

$$
\begin{array}{lll}
\text { if } \theta \text { is atomic } & \text { then } \mathfrak{A} \Vdash \theta & \text { iff } \mathfrak{A} \models \theta \text {; } \\
\text { if } \theta \text { is }(\varphi \vee \psi) & \text { then } \mathfrak{A} \Vdash \theta & \text { iff } \mathfrak{A} \Vdash \varphi \text { or } \mathfrak{A} \Vdash \psi ; \\
\text { if } \theta \text { is } \exists y \varphi(y) & \text { then } \mathfrak{A} \Vdash \theta & \text { iff } \mathfrak{A} \Vdash \varphi(a) \text { for some } a \in A ; \\
\text { if } \theta \text { is } \neg \varphi & \text { then } \mathfrak{A} \Vdash \theta & \text { iff there is no } \mathfrak{B} \in \mathrm{K} \text { such that } \mathfrak{A} \leq \mathfrak{B} \text { and } \\
& & \mathfrak{B} \Vdash \varphi .
\end{array}
$$

Technically our notation should indicate the dependence of forcing on $(K, \leq)$, but we suppress this since the context will make clear what directed family is involved. Note that $\wedge, \rightarrow$, and $\forall$ are treated as defined symbols in the context of forcing. Weak forcing is introduced as usual: $\mathfrak{U} \Vdash^{w} \theta$ if and only if $\mathfrak{U} \Vdash \neg \neg \theta$. Since $(K, \leq)$ will always denote a directed family of finite structures, we will frequently omit this in statements of theorems and definitions.

Before defining forcing generics we need to extend the definition of $\leq$ which we accomplish using the definability condition (4) in the definition of directed family. 
Definition 2.3 Let $\mathfrak{B} \in K$ and let $\mathfrak{M}$ be arbitrary. Then $\mathfrak{B} \leq \mathfrak{M}$ holds if and only if $\mathfrak{B} \subseteq \mathfrak{M}$ and $\mathfrak{M}=\varphi(\bar{b})$ for all $\varphi \in p^{\mathfrak{B}}$, where $\bar{b}$ enumerates $B$.

Definition 2.4 Let $(K, \leq)$ be a directed family and let $\mathfrak{M}$ be arbitrary. $\mathfrak{M}$ is a forcing generic if and only if for every $\theta \in S n_{L(M)}, \mathfrak{M} \models \theta$ if and only if there is some $\mathfrak{U} \in K$ such that $\mathfrak{U} \leq \mathfrak{M}$ and $\mathfrak{U} \Vdash \theta$.

Note that we allow $\mathfrak{M}$ to be uncountable. Accommodating uncountable structures also requires us to extend the notion of $(K, \leq)$-union as follows.

Definition 2.5 Let $(K, \leq)$ be a directed family of finite structures. A structure $\mathfrak{M}$ is a $(K, \leq)$-union if and only if every finite subset $X$ of $M$ is contained in the universe of some $\mathfrak{U} \leq \mathfrak{M}$.

Forcing generic structures then are those obtained from "generic sequences" of structures in $K$.

Lemma 2.6 Let $(K, \leq)$ be a directed family. A structure $\mathfrak{M}$ is a forcing generic if and only if $\mathfrak{M}$ is a $(K, \leq)$-union and for every $\theta \in S n_{L(M)}$ there is some $\mathfrak{A} \leq \mathfrak{M}$ such that $\mathfrak{M} \Vdash(\theta \vee \neg \theta)$.

From the above lemma the existence of forcing generics follows as usual. The existence of a $(K, \leq)$-universal forcing generic uses the countability of $K$ and joint embedding.

Theorem 2.7 Let $(K, \leq)$ be a directed family. Then for every $\mathfrak{A} \in K$ there is a countable forcing generic $\mathfrak{M}$ such that $\mathfrak{U} \leq \mathfrak{M}$. In fact, there is a countable forcing generic $\mathfrak{M}$ which is $(K, \leq)$-universal, that is, every $\mathfrak{U} \in K$ embeds into $\mathfrak{M}$ as a $\leq$-substructure.

The existence of forcing generics has the usual characterization of weak forcing as a consequence.

Corollary 2.8 For every $\mathfrak{A} \in K, \theta \in S n_{L(A)}, \mathfrak{A} \Perp^{w} \theta$ if and only if $\mathfrak{M} \models \theta$ for every forcing generic $\mathfrak{M}$ with $\mathfrak{Q} \leq \mathfrak{M}$.

The following Löwenheim-Skolem result now enables us to construct uncountable forcing generics as unions of families of countable forcing generics. In particular, the union of any elementary chain of forcing generics is forcing generic.

Lemma 2.9 A structure $\mathfrak{M}$ is forcing generic if and only if every finite $X \subseteq M$ is contained in the universe of some countable forcing generic $\mathfrak{N}$ such that $\mathfrak{R} \prec \mathfrak{M}$.

We note the following consequence of joint embedding which enables us to refer to the complete theory of the forcing generics.

Lemma 2.10 Let $(K, \leq)$ be a directed family and assume $\mathfrak{M}$ and $\mathfrak{N}$ are both forcing generic. Then $\mathfrak{M} \equiv \mathfrak{N}$.

Recall that $\mathfrak{M} \subseteq_{\forall} \mathfrak{N}$ means that for all universal formulas $\theta(\bar{x})$ and all $\bar{a} \subseteq M$ we have $\mathfrak{M} \models \theta(\bar{a})$ if and only if $\mathfrak{N}=\theta(\bar{a})$. If $\mathfrak{M}$ and $\mathfrak{N}$ are both forcing generics and $\mathfrak{M} \subseteq \forall \mathfrak{i}$ then $\mathfrak{M} \prec \mathfrak{N}$. In fact, by further extending the definition of $\leq$, we can prove a stronger statement.

Definition 2.11 Let $\mathfrak{M}$ be a $(K, \leq)$-union and assume $\mathfrak{M} \subseteq \mathfrak{N}$. Then $\mathfrak{M} \leq \mathfrak{N}$ holds if and only if for every $\mathfrak{U} \in K$, if $\mathfrak{U} \leq \mathfrak{M}$ then $\mathfrak{A} \leq \mathfrak{A}$. 
Due to the requirement that the formulas in $p^{\mathfrak{A}}$ be universal, we know that $\mathfrak{M} \subseteq \subseteq_{\forall} \mathfrak{A}$ implies $\mathfrak{M} \leq \mathfrak{N}$ for any $(K, \leq$ )-union $\mathfrak{M}$.

Lemma 2.12 Let $\mathfrak{M}, \mathfrak{R}$ be forcing generics. If $\mathfrak{M} \leq \mathfrak{N}$ then $\mathfrak{M} \prec \mathfrak{N}$.

Finally we remark that forcing can be extended to infinitary sentences by adding to the definition the clause $\mathfrak{A} \Vdash \bigvee \Phi$ if and only if $\mathfrak{A} \Vdash \varphi$ for some $\varphi \in \Phi$. If $L^{*}$ is some fragment of $L_{\infty \omega}$ then we can say that $\mathfrak{M}$ is forcing generic with respect to $L^{*}$ if and only if for every $\varphi(\bar{x})$ in $L^{*}$ and $\bar{a} \subseteq M$ we have $\mathfrak{M} \models \varphi(\bar{a})$ if and only if $\mathfrak{U} \Vdash \varphi(\bar{a})$ for some $\mathfrak{U} \leq \mathfrak{M}$.

The existence of forcing generics with respect to countable fragments can then be proved just as Theorem 2.7 (see [3] for more detail). However, there need not be a forcing generic with respect to all of $L_{\omega_{1} \omega}$. Such forcing generics, when they exist, have very nice properties and are the main subject of this paper.

\section{Essential Forcing Generics}

Examples (see the end of this section) show that there may be many countable forcing generics for a given $(K, \leq)$. In this section we show how to pick out a unique, countable forcing generic called an essential forcing generic. Essential forcing generics may be characterized in a way analogous to the definition of $(K, \leq)$-generics and the corresponding existence theorem is also analogous to that concerning $(K, \leq)$ generics.

Definition 3.1 A countable structure $\mathfrak{M} i$ is an essential forcing generic if and only if $\mathfrak{M}$ is forcing generic with respect to all of $L_{\omega_{1} \omega}$.

The uniqueness of essential forcing generics is a consequence of joint embedding and the existence of Scott sentences.

Theorem 3.2 If $\mathfrak{M}$ and $\mathfrak{N}$ are both essential forcing generics, then $\mathfrak{M} \cong \mathfrak{N}$.

Proof: $\quad$ Let $\sigma$ of $L_{\omega_{1} \omega}$ be the Scott sentence of $\mathfrak{M}$. Then $\mathfrak{A} \Vdash \sigma$ for some $\mathfrak{A} \leq \mathfrak{M}$. Since $(K, \leq)$ satisfies joint embedding it follows that there is no $\mathfrak{B} \in K$ such that $\mathfrak{B} \Vdash \neg \sigma$, hence $\mathfrak{B} \Vdash^{w} \sigma$ for all $\mathfrak{B} \in K$. Taking $\mathfrak{B} \leq \mathfrak{N}$, we conclude that $\mathfrak{N} \models \sigma$, hence $\mathfrak{M} \cong \mathfrak{N}$.

Essential forcing generics are precisely the $(K, \leq)$ "homogeneous-universal models" for the following weaker notion of homogeneity.

Definition 3.3 Let $(K, \leq)$ be a directed class. A countable structure $\mathfrak{M}$ is weakly $(K, \leq)$-homogeneous if and only if for every $\mathfrak{U} \leq \mathfrak{M}$ there is some $\mathfrak{B} \in K$ such that $\mathfrak{U} \leq \mathfrak{B} \leq \mathfrak{M}_{i}$ and whenever $f: \mathfrak{B} \cong \mathfrak{B}^{\prime}$ where $\mathfrak{B}^{\prime} \leq \mathfrak{M}$ then $f \uparrow A$ extends to an automorphism of $\mathfrak{M}$.

Thus, the notion of $(K, \leq)$-homogeneous from [4] is the strengthening of this weak form in which $\mathfrak{B}=\mathfrak{A}$.

To prove our characterization of essential forcing generics we require the following lemma, whose proof is a standard back-and-forth argument which is omitted.

Lemma 3.4 Let $\mathfrak{M}$ be a countable $(K, \leq)$-universal $(K, \leq)$-union. Then $\mathfrak{M}$ is weakly $(K, \leq)$-homogeneous if and only if the following holds: 
for every $\mathfrak{A} \leq \mathfrak{M}$ there is some $\mathfrak{B}$ such that $\mathfrak{A} \leq \mathfrak{B} \leq \mathfrak{M}$ and whenever $\mathfrak{S} \in K, \mathfrak{B} \leq \mathfrak{C}$ then there is some $f: \mathfrak{C} \cong \mathfrak{C}^{\prime}$ where $\mathfrak{S}^{\prime} \leq \mathfrak{M}$ and $f \uparrow A=i d_{A}$.

If the countable structure $\mathfrak{M}$ is a $(K, \leq)$-union and is weakly $(K, \leq)$-homogeneous then for every $\bar{a} \subseteq M$ there is some $\mathfrak{B} \in K, \bar{a} \subseteq B$, and $\mathfrak{B} \leq \mathfrak{M}$ such that whenever $f: \mathfrak{B} \cong \mathfrak{B}^{\prime}$ where $\mathfrak{B}^{\prime} \leq \mathfrak{M}$ then $f \mid \bar{a}$ extends to an automorphism of $\mathfrak{M}$. This follows by finding $\mathfrak{A} \in K$ with $\bar{a} \subseteq A$ and $\mathfrak{U} \leq \mathfrak{M}$ and then applying the definition of weakly $(K, \leq)$-homogeneous. This same comment holds also for the condition $3^{*}$ in Lemma 3.4.

Theorem 3.5 A countable structure $\mathfrak{M}$ is an essential forcing generic if and only if the following hold:

1. Mi is a $(K, \leq)$-union,

2. $\mathfrak{M}$ is $(K, \leq)$-universal,

3. $\mathfrak{M}$ is weakly $(K, \leq)$-homogeneous.

Proof: First we show that (1), (2), and (3) will hold assuming $\mathfrak{M}$ is an essential forcing generic. (1) holds since all forcing generics are $(K, \leq)$-unions. To show (2), let $\mathfrak{X} \in K$ and let $\sigma$ be $\exists \bar{x} \varphi(\bar{x})$ where $\varphi(\bar{x})=\bigwedge p^{\mathfrak{A}}(\bar{x})$. Then $\mathfrak{A} \Vdash \sigma$, since $\mathfrak{B} \models \varphi(\bar{a})$ for all $\mathfrak{B} \in K$ with $\mathfrak{A} \leq \mathfrak{B}$. Hence, by joint embedding, $\mathfrak{B} \Vdash^{w} \sigma$ for all $\mathfrak{B} \in K$. Therefore, $\mathfrak{M} \models \sigma$ and so $\mathfrak{U} \cong \mathfrak{U}^{\prime}$ for some $\mathfrak{U}^{\prime} \leq \mathfrak{M}$, since we may assume that $p^{\mathfrak{I}}$ contains the open diagram of $\mathfrak{A}$.

To show that (3) holds, fix some $\mathfrak{U} \leq \mathfrak{M}$ and let $\bar{a}$ list the elements of $A$. There is then a Scott formula $\varphi_{\bar{a}}(\bar{x})$ in $L_{\omega_{1} \omega}$ such that for all $\bar{a}^{\prime} \subseteq M$ we have $\mathfrak{M}=\varphi_{\bar{a}}\left(\overline{a^{\prime}}\right)$ if and only if $(\mathfrak{M}, \bar{a}) \cong\left(\mathfrak{M}, \bar{a}^{\prime}\right)$. Choose $\mathfrak{B}$ so that $\mathfrak{A} \leq \mathfrak{B} \leq \mathfrak{M}$ and $\mathfrak{B} \Vdash \varphi_{\bar{a}}(\bar{a})$. Then $\mathfrak{B}$ is as required in the definition of weak $(K, \leq)$-homogeneity, since if $f: \mathfrak{B} \cong \mathfrak{B}^{\prime}$ where $\mathfrak{B}^{\prime} \leq \mathfrak{M}$ then $\mathfrak{B}^{\prime} \Vdash \varphi_{\bar{a}}(f(\bar{a}))$. Hence $(\mathfrak{M}, \bar{a}) \cong(\mathfrak{M}, f(\bar{a}))$ by the choice of $\varphi_{\bar{a}}$.

For the other direction, we assume $\mathfrak{M}$ is countable and satisfies (1), (2), and (3). We show directly by induction on $\theta(\bar{x})$ of $L_{\omega_{1} \omega}$ that for every $\bar{a} \subseteq M, \mathfrak{M}=\theta(\bar{a})$ if and only if $\mathfrak{B} \Vdash \theta(\bar{a})$ for some $\mathfrak{B} \leq \mathfrak{M}$. The only case requiring argument is $\theta(\bar{a})=\neg \varphi(\bar{a})$. By the inductive hypothesis, $\mathfrak{M} \models \theta(\bar{a})$ if and only if there is no $\mathfrak{B} \leq \mathfrak{M}$ such that $\mathfrak{B} \Vdash \varphi(\bar{a})$. In particular, if there is some $\mathfrak{B} \leq \mathfrak{M}$ such that $\mathfrak{B} \Vdash \theta(\bar{a})$, then $\mathfrak{M} \models \theta(\bar{a})$. For the converse, assume $\mathfrak{M} \models \theta(\bar{a})$. By the remark after Lemma 3.4, we may choose $\mathfrak{B}$ as in ( $\left.3^{*}\right)$ of Lemma 3.4 so that $\bar{a} \subseteq B$. We claim that $\mathfrak{B} \Vdash \theta(\bar{a})$. If not, then there is some $\mathfrak{S} \in K$ such that $\mathfrak{B} \leq \mathfrak{C}$ and $\mathfrak{C} \Vdash \varphi(\bar{a})$. But then, by Lemma 3.4, there is $f: \mathfrak{C} \cong \mathbb{C}^{\prime}$ where $\mathfrak{S}^{\prime} \leq \mathfrak{M}$ and $f\left\lceil\bar{a}=i d_{\bar{a}}\right.$. Thus $\tilde{C}^{\prime} \Vdash \varphi(\bar{a})$, contradicting the inductive hypothesis.

$(K, \leq)$-generics are thus seen to be essential forcing generics. The following result characterizes $(K, \leq)$-generics completely in terms of forcing.

Theorem 3.6 Let $(K, \leq)$ be a directed family. If $\mathfrak{M}$ is $(K, \leq)$-generic then $\mathfrak{M}$ is an essential forcing generic. Moreover, $\mathfrak{M}$ is $(K, \leq)$-generic if and only if for every formula $\varphi(\bar{x})$ of $L_{\omega_{1} \omega}$ and every $\bar{a} \in M, \mathfrak{M} \models \varphi(\bar{a})$ if and only if $\mathfrak{B} \Vdash^{w} \varphi(\bar{a})$ for every $\mathfrak{B} \leq \mathfrak{M}$ with $\bar{a} \subseteq B$.

Proof: Assume $\mathfrak{M}$ is $(K, \leq)$-generic. The proof that $\mathfrak{M} \models \varphi(\bar{a})$ if and only if $\mathfrak{B} \Vdash^{w} \varphi(\bar{a})$ for every $\mathfrak{B} \leq \mathfrak{M}$ with $\bar{a} \subseteq B$ is by induction. It is exactly like the 
last paragraph of the proof of Theorem 3.5, except that (strong) $(K, \leq)$-homogeneity yields the right-hand side for all such $\mathfrak{B}$.

For the other direction it suffices to show $(K, \leq)$-homogeneity holds. This too is just like the corresponding part of the proof of Theorem 3.5.

$(K, \leq)$-generics are also universal among forcing generics. The proof of this requires the following lemma, implicit in the proof of Proposition 3.1 of [4].

Lemma 3.7 If $\mathfrak{M}$ is $(K, \leq)$-generic and $\mathfrak{N}$ is a countable $(K, \leq)$-union, then $\mathfrak{N} \cong \mathfrak{N}^{\prime}$ for some $\mathfrak{N}^{\prime} \leq \mathfrak{M}$.

Part (b) of the next corollary is an interesting consequence of the universality of $(K, \leq)$-generics given in part (a).

Corollary 3.8 Let $\mathfrak{M}$ be $(K, \leq)$-generic and let $T=T h(\mathfrak{M})$.

(a) A countable structure $\Re$ is forcing generic if and only if $\Re$ is a $(K, \leq)$-union and $\mathfrak{N}$ is elementarily embeddable in $\mathfrak{M}$.

(b) If every countable model of $T$ can be elementarily embedded in some forcing generic, then $\mathfrak{M}$ is $\omega$-saturated.

Proof: The left to right direction of (a) is immediate from Lemma 3.7, Theorem 3.6 and Lemma 2.12. For the other direction, assume $\mathfrak{N}$ is a $(K, \leq)$-union and $\mathfrak{N} \prec \mathfrak{M}$. We show by induction on formulas $\theta(\bar{x})$ of $L$ that for all $\bar{a} \subseteq N$ we have $\mathfrak{R} \models \theta(\bar{a})$ if and only if $\mathfrak{U} \Vdash \theta(\bar{a})$ for some $\mathfrak{U} \leq \mathfrak{N}$.

Since $\Re$ is a $(K, \leq)$-union, all cases are trivial except for $\theta=\neg \varphi$. Assuming $\mathfrak{N} \equiv \theta(\bar{a})$, we in fact show that $\mathfrak{U} \Vdash \theta(\bar{a})$ for every $\mathfrak{U} \leq \mathfrak{N}$ with $\bar{a} \subseteq A$. Given such an $\mathfrak{A}$, suppose that $\mathfrak{U} \leq \mathfrak{B}$ for some $\mathfrak{B} \in K$ with $\mathfrak{B} \Vdash \varphi(\bar{a})$. Then there is such a $\mathfrak{B} \leq \mathfrak{M}$ because $\mathfrak{M}$ is $(K, \leq)$-generic. Hence, $\mathfrak{M} \models \varphi(\bar{a})$, contradicting the hypothesis that $\mathfrak{N} \prec \mathfrak{M}$.

By part (a) and Lemma 2.9, every countable model of $T$ can be elementarily embedded in $\mathfrak{M}$. Therefore, $\mathfrak{M}$ is $\omega$-saturated by Proposition 3.1 of [4] or by its generalization, Lemma 4.5 of the following section.

There are examples (see below) of directed classes $(K, \leq)$ for which no essential forcing generic exists. What is needed in addition is a weak version of amalgamation, just as full amalgamation is what is needed for the existence of $(K, \leq)$-generics. We omit the proof, which is similar to the proof of the corresponding result for $(K, \leq)$ generics.

Definition 3.9 A directed class $(K, \leq)$ satisfies the weak amalgamation property if and only if for every $\mathfrak{U} \in K$ there is some $\mathfrak{D} \in K$ such that $\mathfrak{U} \leq \mathfrak{D}$ and whenever $\mathfrak{D} \leq \mathfrak{B}_{0}, \mathfrak{B}_{1}$ then there is $\mathfrak{E} \in K$ and isomorphic embeddings $f_{i}: \mathfrak{B}_{i} \rightarrow \mathfrak{E}$ so that $f_{i}\left(\mathfrak{B}_{i}\right) \leq \mathfrak{C}$ for $i=1,2$ and $f_{1}\left\lceil A=f_{2}\lceil A\right.$.

Theorem 3.10 Let $(K, \leq)$ be a directed class of finite structures. Then there is an essential forcing generic if and only if $(K, \leq)$ satisfies weak amalgamation.

By exploiting the forcing definition of essential forcing generics, we obtain the following useful sufficient condition for their existence.

Lemma 3.11 Let $(K, \leq)$ be a directed class and assume there are only countably many countable forcing generics up to isomorphism. Then there is an essential forcing generic. 
Proof: Let $\left\{\sigma_{n}: n \in \omega\right\}$ list Scott sentences for every countable forcing generic. Then $\bigvee_{n \in \omega} \sigma_{n}$ is true on every countable forcing generic, hence is weakly forced by every $\mathfrak{A} \in K$. So there is some $n \in \omega$ and some $\mathfrak{A} \in K$ such that $\mathfrak{A} \Vdash \sigma_{n}$. Let $\mathfrak{M}$ be the countable model whose Scott sentence is $\sigma_{n}$. If $L^{*}$ is any countable fragment of $L_{\omega_{1} \omega}$ containing $\sigma_{n}$ and $\mathfrak{A} \leq \mathfrak{N}$, where $\mathfrak{N}$ is forcing generic with respect to $L^{*}$, then $\mathfrak{M} \cong \mathfrak{R}$. Since there are such forcing generics $\mathfrak{N}$ for every countable fragment of $L_{\omega_{1} \omega}$, it follows that $\mathfrak{M}$ must be forcing generic with respect to each countable fragment and hence must be an essential forcing generic.

We conclude this section with a number of examples.

Example 3.12 The only nonlogical symbol of $L$ is a binary relation $S . \quad K$ is the class of all finite $L$-structures embeddable in $(\mathbb{Z}, S)$, where $S$ is the immediate successor relation on $\mathbb{Z}$. For $\mathfrak{A}, \mathfrak{B} \in K$ we define $\mathfrak{A} \leq \mathfrak{B}$ to hold if and only if $\mathfrak{A} \subseteq \mathfrak{B}$ and for every $a_{1}, a_{2} \in A$ if there is an $S$-chain in $B$ connecting $a_{1}$ to $a_{2}$ then this $S$-chain already belongs to $A$. Then $(K, \leq)$ is a directed family of finite structures, and every model of $T=T h((\mathbb{Z}, S))$ is forcing generic. The countable $\omega$-saturated model of $T$ is the $(K, \leq)$-generic.

Example 3.13 $L, K$, and $T$ are as in the above example, but the extension relation on $K$ is ordinary substructure. Then once again every model of $T$ is forcing generic, but there is no $(K, \subseteq)$-generic since amalgamation fails. However, $(\mathbb{Z}, S)$ is an essential forcing generic, since every structure in $K$ forces $\forall x \forall y[(x \neq$ $\left.y) \rightarrow \bigvee_{n \in \omega}\left[\varphi_{n}(x, y) \vee \varphi_{n}(y, x)\right]\right]$ where $\varphi_{0}(x, y)$ is $S(x, y)$ and $\varphi_{n+1}(x, y)$ is $\exists u_{0}, \ldots, u_{n}\left[S\left(x, u_{0}\right) \wedge \cdots \wedge S\left(u_{n}, y\right)\right]$. This follows because any pair of elements of $A$ can be connected in some $\mathfrak{B}$ with $\mathfrak{A} \subseteq \mathfrak{B}$.

Example 3.14 $L$ and $T$ are as in the above examples. $K^{\prime}$ is the class of finite $L$-structures embeddable in $(\mathbb{Z}, S)$ in which every pair of elements is connected by an $S$-chain and the extension relation is ordinary substructure. Then $\left(K^{\prime}, \subseteq\right)$ is directed. $(\mathbb{Z}, S)$ is the only forcing generic and is $\left(K^{\prime}, \subseteq\right)$-generic.

Example 3.15 $L$ is as in the previous examples and $L^{*}$ adds a unary predicate $P$. $K^{*}$ is the class of all finite $L^{*}$-structures $\mathfrak{I}^{*}$ so that $\mathfrak{H}^{*} \backslash L \in K$, from Example 3.12. We define $\leq^{*}$ on $K^{*}$ by $\mathfrak{A}^{*} \leq * \mathfrak{B}^{*}$ if and only if $\mathfrak{A}^{*} \subseteq \mathfrak{B}^{*}$ and $\mathfrak{A}^{*} \uparrow L \leq \mathfrak{B}^{*} \uparrow L$, where $\leq$ is as in Example 3.12. Then $\left(K^{*}, \leq^{*}\right)$ is directed and all models of the complete theory of the forcing generics are forcing generics. But there is no essential forcing generic, since weak amalgamation fails.

In Example 3.13 we saw that $(\mathbb{Z}, S)$ was an essential forcing generic for $(K, \subseteq)$ which was not $(K, \subseteq)$-generic. However, we showed in Example 3.14 that $(\mathbb{Z}, S)$ was $\left(K^{\prime}, \subseteq\right)$-generic for a suitable choice of $K^{\prime}$. Can this always be done? Does every essential forcing generic become $\left(K^{\prime}, \leq^{\prime}\right)$-generic for some directed family $\left(K^{\prime}, \leq^{\prime}\right)$ ? As the following example shows, the answer is no.

Example 3.16 Let $L=\{\leq, P\}$, where $P$ is a unary "coloring" of points. Let $\Sigma$ be the set of $\forall \exists$ sentences expressing the following:

1. $\leq$ is a tree order of the universe.

2. The tree has one component.

3. All immediate successors of a point have the same color.

4. The least element and its immediate successors all satisfy $P$. 
Let $K$ be the collection of finite models of $\Sigma$. For $\mathfrak{A}, \mathfrak{B} \in K$, we define $\mathfrak{A} \leq \mathfrak{B}$ to hold if and only if $\mathfrak{Q} \subseteq \mathfrak{B}$ and no element of $(B-A)$ precedes any element of $A$. Then $(K, \leq)$ is directed.

There is just one forcing generic $\mathfrak{M}$. It is a model of $\Sigma$; it has a least element and every other element has an immediate predecessor; every element has infinitely many immediate successors (all of the same color), but there are infinitely many of these successors having immediate successors of each color; the tree is well founded of height $\omega$. Since there is only one forcing generic, it is essential by Lemma 3.11.

Claim 3.17 There is no directed $\left(K^{\prime}, \leq^{\prime}\right)$ so that $\mathfrak{M}$ is $\left(K^{\prime}, \leq^{\prime}\right)$-generic.

Proof: It suffices to show that for every $\mathfrak{A} \subseteq \mathfrak{M}(\bar{a}=A)$, which contains some element of height $\leq 2$, there is some $\mathfrak{\mathfrak { H } ^ { \prime }} \subseteq \mathfrak{M}\left(\bar{a}^{\prime}=A^{\prime}\right)$ so that $(\mathfrak{M}, \bar{a}) \equiv_{\forall}\left(\mathfrak{M}, \bar{a}^{\prime}\right)$ but $(\mathfrak{M}, \bar{a}) \not\left(\mathfrak{M}, \bar{a}^{\prime}\right)$. Given such an $\mathfrak{A}$, let $a_{0}$ be a maximal element in $A$ and let $b$ be its immediate predecessor. Choose $a_{0}^{\prime}$ to be an immediate successor of $b$ not in $A$ whose immediate successors all have color different from that of the immediate successors of $a_{0}$. Let $A^{\prime}=\left(A-\left\{a_{0}\right\}\right) \cup\left\{a_{0}^{\prime}\right\}$.

There is an obvious generalization of essential forcing generics to allow uncountable models. $\mathfrak{M}$ is an essential forcing generic if and only if for every formula $\theta(\bar{x})$ of $L_{\infty \omega}$ and every $\bar{a} \subseteq M, \mathfrak{M} \models \theta(\bar{a})$ if and only if there is some $\mathfrak{A} \leq \mathfrak{M}$ such that $\mathfrak{U} \Vdash \theta(\bar{a})$. However, since $K$ is assumed to be countable up to isomorphism, a Löwenheim-Skolem argument shows that such models are precisely the $L_{\infty \omega}$-elementary extensions of countable essential forcing generics. Thus, nothing essentially new is obtained.

\section{Properties of Essential Forcing Generics}

In this section we show that many of properties of $(K, \leq)$-generics established in [ 4 ] are in fact consequences of properties of essential forcing generics.

The first group of results concerns the special case in which $L$ is finite and $p^{\mathfrak{A}}$ is finite for every $\mathfrak{U} \in K$. Under these hypotheses the $(K, \leq)$-generic is atomic (Proposition 3.4 in [4]) and as a consequence one obtains a result characterizing when its complete theory is $\omega$-categorical (Theorem 3.5 of [4]). Both these results, and more, hold for essential forcing generics.

Theorem 4.1 Assume $L$ is finite and $p^{\mathfrak{A}}$ is finite for all $\mathfrak{A} \in K$.

(a) If $\mathfrak{M}$ is an essential forcing generic then $\mathfrak{M}$ is atomic.

(b) There is an essential forcing generic if and only if there is an atomic forcing generic.

Proof: Since $L$ is finite, we may assume that $p^{\mathfrak{A}}$ includes the basic open diagram of $\mathfrak{A}$.

(a) Assume $\mathfrak{M}$ is an essential forcing generic and let $\bar{a} \subseteq M$. By the remark after Lemma 3.4, we may choose $\mathfrak{B}$ so that $\bar{a} \subseteq B$ and $\mathfrak{B}$ satisfies the definition of weak $(K, \leq)$-homogeneity for $\bar{a}$. Let $\varphi(\bar{x})=\exists \bar{y} \wedge p^{\mathfrak{B}}(\bar{x}, \bar{y})$, where $\bar{x}$ corresponds to $\bar{a}$ in the enumeration of $B$. Then $\varphi$ determines $\bar{a}$ up to isomorphism in $\mathfrak{M}$, so it isolates the complete type of $\bar{a}$. Thus $\mathfrak{M}$ is atomic.

(b) It suffices to show that if $\mathfrak{M}$ is an atomic forcing generic then $\mathfrak{M}_{\text {in }}$ is essential forcing generic. We show the conditions in Theorem 3.5 all hold. Certainly 
$\mathfrak{M}$ is a $(K, \leq)$-union. If $\mathfrak{A} \in K$, then $\mathfrak{B} \Vdash^{w} \exists \bar{x} \wedge p^{\mathfrak{A}}(\bar{x})$ for every $\mathfrak{B} \in K$, implying $\mathfrak{M} \models \exists \bar{x} \wedge p^{\mathfrak{A}}(\bar{x})$. Hence, $\mathfrak{A}$ embeds strongly into $\mathfrak{M}$ and $\mathfrak{M}$ is $(K, \leq)$-universal. To show $\mathfrak{M}$ is weakly $(K, \leq)$-homogeneous, let $\mathfrak{A} \leq \mathfrak{M}$ and let $\varphi(\bar{x})$ isolate the complete type of $\bar{a}$ in $\mathfrak{M}$, where $\bar{a}$ enumerates $A$. Then $\mathfrak{B} \Vdash \varphi(\bar{a})$ for some $\mathfrak{B}$ where $\mathfrak{A} \leq \mathfrak{B} \leq \mathfrak{M}$. If $f: \mathfrak{B} \cong \mathfrak{B}^{\prime}$ where $\mathfrak{B}^{\prime} \leq \mathfrak{M}$ then $\mathfrak{M} \models \varphi(f(\bar{a}))$, hence $f\lceil A$ extends to an automorphism of $\mathfrak{M}$ as required.

The following is an immediate consequence of Theorem 4.1.

Corollary 4.2 Assume $L$ is finite and $p^{\mathfrak{A}}$ is finite for all $\mathfrak{A} \in K$. Assume $\mathfrak{M}$ is an essential forcing generic and let $T=T h(\mathfrak{M})$. Then the following are equivalent:

1. $T$ is $\omega$-categorical;

2. $\mathfrak{M}$ is $\omega$-saturated.

The next result has no finiteness assumptions. Note that if $\leq$ is just usual substructure then the hypothesis is precisely that $\mathfrak{M}$ is algebraically prime in the sense of Baldwin and Kueker [1].

Theorem 4.3 Let $\mathfrak{M}$ be an essential forcing generic and assume $T=T h(\mathfrak{M})$ has a prime model. Assume that $\mathfrak{M}$ can be embedded as a strong substructure in every model of T. Then $\mathfrak{M}$ is prime.

Proof: Let $\mathfrak{i}$ be the prime model of $T$. Then by assumption $\mathfrak{M} \cong \mathfrak{N}^{\prime} \leq \mathfrak{N}$. It suffices to show $\mathfrak{N}$ is forcing generic, since we then have $\mathfrak{M}^{\prime} \prec \mathfrak{N}$ by Lemma 2.12, and thus $\mathfrak{M}$ is prime.

We may assume $\mathfrak{N} \prec \mathfrak{M}$. It suffices to show that if $\mathfrak{N} \models \theta(\bar{a})$ then there is some $\mathfrak{C} \leq \mathfrak{N}$ such that $\mathfrak{C} \Vdash \theta(\bar{a})$. Let $\varphi(\bar{a})$ isolate the type of $\bar{a}$ in $\mathfrak{N}$, hence in $\mathfrak{M}$. Then $\mathfrak{M}=\theta(\bar{a}) \wedge \varphi(\bar{a})$ implying $\mathfrak{A} \Vdash \theta(\bar{a})$ and $\mathfrak{A} \Vdash \varphi(\bar{a})$ for some $\mathfrak{A} \leq \mathfrak{M}$. But $\mathfrak{M} \cong \mathfrak{M}^{\prime}$ for some $\mathfrak{M}^{\prime} \leq \mathfrak{N}$. So there is some $\mathfrak{U}^{\prime} \cong \mathfrak{U}, \mathfrak{H}^{\prime} \leq \mathfrak{M}^{\prime} \leq \mathfrak{A}$ and $\mathfrak{H}^{\prime} \Vdash \theta\left(\bar{a}^{\prime}\right), \mathfrak{Y}^{\prime} \Vdash \varphi\left(\bar{a}^{\prime}\right)$ where $\bar{a}^{\prime}$ is the image of $\bar{a}$ under the isomorphism. But $\mathfrak{N} \prec \mathfrak{M}$ so $\mathfrak{U}^{\prime} \leq \mathfrak{M}$ and $\mathfrak{M} \models \varphi\left(\bar{a}^{\prime}\right) \wedge \theta\left(\bar{a}^{\prime}\right)$, hence $\mathfrak{N} \models \varphi\left(\bar{a}^{\prime}\right) \wedge \theta\left(\bar{a}^{\prime}\right)$. Thus, $(\mathfrak{N}, \bar{a}) \cong\left(\mathfrak{i}, \bar{a}^{\prime}\right)$. The inverse image of $\mathfrak{V}^{\prime}$ under the isomorphism will thus be some $\mathfrak{B}$ such that $\mathfrak{B} \leq \mathfrak{M}$ and $\mathfrak{B} \Vdash \theta(\bar{a})$, as desired.

The main results in [4] concern the circumstances under which the $(K, \leq)$-generic is $\omega$-saturated. In particular, the $(K, \leq)$-generic is $\omega$-saturated provided it is weakly saturated (Proposition 3.1 of [4]), and in that case its complete theory is 1-model complete (Theorem 3.2 of [4]). We establish the same facts for essential forcing generics as consequences of some more general results. We recall the definition of $\omega$-homogeneity.

Definition 4.4 A structure $\mathfrak{M}$ is $\omega$-homogeneous if and only if for any $\bar{a}, \bar{b} \subset M$, if $(\mathfrak{M}, \bar{a}) \equiv(\mathfrak{M}, \bar{b})$ then for every $c$ there is a $d$ such that $(\mathfrak{M}, \bar{a}, c) \equiv(\mathfrak{M}, \bar{b}, d)$.

Examples show that an essential forcing generic need not be $\omega$-homogeneous but the following result explains the limitation.

Lemma 4.5 Assume $\mathfrak{M}$ is an essential forcing generic and that there is some $\omega$-homogeneous model $\mathfrak{X}$ realizing precisely the same types as $\mathfrak{M}$. Then $\mathfrak{M}$ is $\omega$ homogeneous. 
Proof: Let $\mathfrak{M}, \mathfrak{R}$ be as in the statement, assume $(\mathfrak{M}, \bar{a}) \equiv(\mathfrak{M}, \bar{b})$ and let $c \in M$ be given. We show that there is some $d \in M$ such that $(\mathfrak{M}, \bar{a}, c) \equiv(\mathfrak{M}, \bar{b}, d)$. By the remark after Lemma 3.4, we may assume $\bar{b} \subseteq C$ where $\mathfrak{S} \leq \mathfrak{M}$ and $\mathfrak{S}$ satisfies the definition of weak $(K, \leq)$-homogeneity for $\bar{b}$. Since $\mathfrak{N}$ realizes all types realized in $\mathfrak{M}$, we can find $\bar{a}^{*}, c^{*}, \bar{b}^{*}=B^{*}$ and $\mathfrak{S}^{*}$ such that $\left(\Re, \bar{a}^{*}\right) \equiv\left(\Re, \bar{b}^{*}\right)$, $\mathfrak{C}^{*} \leq \mathfrak{N}$, and $(\mathfrak{C}, \bar{b}) \cong\left(\mathfrak{\complement}^{*}, \bar{b}^{*}\right)$. Since $\mathfrak{N}$ is $\omega$-homogeneous, there is some $d^{*} \in N$ so that $\left(\Re, \bar{a}^{*}, c^{*}\right) \cong\left(\Re, \bar{b}^{*}, d^{*}\right)$. Now, since $\mathfrak{M}$ realizes all types realized in $\Re$, we can find $\bar{b}^{\prime}, d^{\prime}$, $\mathfrak{C}^{\prime}$ such that $\left(\mathfrak{M}, \bar{b}^{\prime}, d^{\prime}\right) \equiv\left(\mathfrak{N}, \bar{b}^{*}, d^{*}\right), \mathfrak{C}^{\prime} \leq \mathfrak{M}$, and $\left(\mathfrak{C}^{*}, \bar{b}^{*}\right) \cong$ $\left(\mathfrak{S}^{\prime}, \bar{b}^{\prime}\right)$, that is, $(\mathfrak{S}, \bar{b}) \cong\left(\mathbb{S}^{\prime}, \bar{b}^{\prime}\right)$. By the choice of $\mathfrak{S}$ the restriction of this last isomorphism to $\bar{b}$ extends to an automorphism of $\mathfrak{M}$ and the inverse image of $d^{\prime}$ will be the desired $d$.

The following is an immediate consequence.

Theorem 4.6 If $\mathfrak{M}$ is an essential forcing generic and weakly saturated (i.e., realizes all pure types consistent with its complete theory), then $\mathfrak{M}_{\text {is }} \omega$-saturated.

Without reference to essential forcing generics, we show that the complete theory $T$ of the forcing generics is 1-model complete, assuming every type consistent with $T$ is realized on some forcing generic. We first note the following.

Lemma 4.7 Let $T$ be the complete theory of the forcing generics. Assume every type consistent with $T$ is realized in some forcing generic. Then every $\omega$-saturated model of $T$ is forcing generic.

Proof: Let $\mathfrak{M}$ be an $\omega$-saturated model of $T$ (possibly uncountable) and let $\bar{a} \subseteq M$. By hypothesis the complete type of $\bar{a}$ is realized on some forcing generic $\mathfrak{A}$ which we may take to be countable. Since $\mathfrak{M}$ is $\omega_{1}$-universal, we may also take $\mathfrak{N} \prec \mathfrak{M}$. Thus, $\mathfrak{M}$ is also forcing generic by Lemma 2.9 .

We now obtain the following result giving a sufficient condition for the complete theory of the forcing generics to be 1-model complete.

Theorem 4.8 Let $T$ be the complete theory of the forcing generics. Assume that every type consistent with $T$ is realized in some forcing generic. Then $T$ is 1-model complete. If $\leq$ is ordinary substructure, then $T$ is model complete.

Proof: Let $\mathfrak{M}$ be an $\omega$-saturated model of $T$. By Lemma $4.7 \mathfrak{M}$ is forcing generic. If $T$ is not 1-model complete, then there are $\mathfrak{N}, \mathfrak{N}^{\prime} \models T$ such that $\mathfrak{N} \subseteq \forall \mathfrak{N}^{\prime}$ but $\mathfrak{N} \nprec \mathfrak{R}^{\prime}$. So there is $\bar{a} \subseteq N$ such that $(\mathfrak{N}, \bar{a}) \Rightarrow_{\exists \forall}\left(\mathfrak{N}^{\prime}, \bar{a}\right)$ but $(\mathfrak{N}, \bar{a}) \not \equiv\left(\mathfrak{N}^{\prime}, \bar{a}\right)$. Since $\mathfrak{M}$ is saturated, we get $\bar{b}, \bar{b}^{\prime} \subseteq M$ such that $(\mathfrak{M}, \bar{b}) \equiv(\mathfrak{R}, \bar{a})$ and $\left(\mathfrak{M}, \bar{b}^{\prime}\right) \equiv\left(\mathfrak{N}^{\prime}, \bar{a}\right)$ hence $(\mathfrak{M}, \bar{b}) \Rightarrow_{\exists \forall}\left(\mathfrak{M}, \bar{b}^{\prime}\right)$ but $(\mathfrak{M}, \bar{b}) \not \equiv\left(\mathfrak{M}, \bar{b}^{\prime}\right)$.

Now, extend $\bar{b}$ to some $C$ where $\mathfrak{S} \in K$, $\mathfrak{S} \leq \mathfrak{M}$. Since $\mathfrak{M}$ is $\omega$-saturated, there is $C^{\prime}$ such that $(\mathfrak{M}, \bar{b}, \bar{c} \backslash \bar{b}) \Rightarrow \forall\left(\mathfrak{M}, \bar{b}^{\prime}, \bar{c}^{\prime} \backslash \bar{b}^{\prime}\right)$ where $\bar{c}, \bar{c}^{\prime}$ enumerate $C, C^{\prime}$, respectively. Therefore, $(\mathfrak{C}, \bar{b}) \cong\left(\mathfrak{S}^{\prime}, \bar{b}^{\prime}\right)$ and $\mathfrak{S}^{\prime} \leq \mathfrak{M}$, hence $(\mathfrak{M}, \bar{b}) \equiv\left(\mathfrak{M}, \bar{b}^{\prime}\right)$ which is a contradiction. In the case in which $\leq$ is $\subseteq$, the same argument shows model completeness.

In the special case in which $\leq$ is ordinary substructure, [4] obtains a more satisfactory result characterizing the circumstances under which the $(K, \leq)$-generic is $\omega$-saturated (Theorem 2.5 of [4]). We generalize this result to essential forcing generics satisfying one more condition. One of the main distinctions between $(K, \leq)$-generics 
and essential forcing generics, as shown in Example 3.13, is that an essential forcing generic need not embed every countable $(K, \leq)$-union. If we add this property then we obtain the exact analogue to Theorem 2.5 of [4].

We first need the following generalization of Lemma 2.4 of [4].

Lemma 4.9 Assume $\leq$ is ordinary substructure. Let $T$ be the complete theory of the forcing generics and assume every countable model of $T$ embeds in some $(K, \subseteq)$-union. If $\mathfrak{M}$ is an essential forcing generic, then $\mathfrak{M}$ is an existentially closed model of $T_{\forall}$.

Proof: Assume $\theta(\bar{x}, \bar{y})$ is an open formula, $\bar{a} \in M$, and $\mathfrak{l}=\exists \bar{x} \theta(\bar{x}, \bar{a})$ for some $\mathfrak{N}$ such that $\mathfrak{N} \models T_{\forall}$ and $\mathfrak{M} \subseteq \mathfrak{N}$. Since $\mathfrak{N}$ is embeddable in a model of $T$, and by the hypothesis of Lemma 4.9, we may assume $\mathfrak{N}$ is a $(K, \subseteq)$-union. By adding dummy variables as needed, it may be assumed that $\bar{a}$ is the universe of some $\mathfrak{A} \in K, \mathfrak{H} \subseteq \mathfrak{M}$. Since weak amalgamation holds for $(K, \subseteq)$, there is $\mathfrak{B} \in K, \mathfrak{B} \supseteq \mathfrak{U}$ such that any two extensions of $\mathfrak{B}$ can be amalgamated over $\bar{a}$, that is, $\mathfrak{B}$ satisfies weak amalgamation for $\mathfrak{U}$. Let $\mathfrak{S} \subseteq \mathfrak{N}$ be finite with $\mathfrak{S} \models \exists \bar{x} \theta(\bar{x}, \bar{a})$ and $\mathfrak{B} \subseteq \mathfrak{S}$. Since $\mathfrak{N}$ is a $(K, \subseteq)$-union, there is $\mathfrak{S}^{\prime} \in K, \mathfrak{C} \subseteq \mathfrak{C}^{\prime}$. Now weak amalgamate $\mathfrak{C}^{\prime}$ into $\mathfrak{M}$ over $\bar{a}$.

Finally we obtain the following.

Theorem 4.10 Assume $\leq$ is ordinary substructure. Let $M$ d be an essential forcing generic and assume every countable $(K, \subseteq)$-union embeds into $\mathfrak{M}$. Let $T=T h(\mathfrak{M})$. Then the following are equivalent:

1. Mis $\omega$-saturated;

2. $T$ is model complete and every countable model of $T$ embeds into some ( $K, \subseteq$ )-union;

3. $T_{\forall}$ has a model companion and every countable model of $T$ embeds into some $(K, \subseteq)$-union.

Proof: $\quad(1 \Rightarrow 2) \quad$ Assume that $\mathfrak{M}$ is $\omega$-saturated. Then any countable $\mathfrak{N}$ which models $T$ embeds (elementarily) into $\mathfrak{M}$, which is a $(K, \subseteq)$-union. Since the essential forcing generic is $\omega$-saturated, $T$ is model complete by Theorem 4.8 .

( $2 \Rightarrow 3$ ) Since $T$ is model complete, $T$ is the model companion of $T_{\forall}$.

$(3 \Rightarrow 1) \quad$ Assume that $S$ is the model companion of $T_{\forall} . \mathfrak{M}$ is an existentially closed model of $T_{\forall}=S_{\forall}$ by Lemma 4.9. Thus $\mathfrak{M} \models S$ so $T=S$ is model complete. By hypothesis, however, every countable model of $T$ embeds isomorphically, hence elementarily, into $\mathfrak{M}$. Hence, $\mathfrak{M}$ is a weakly saturated model of $T$, so $\mathfrak{M}$ is $\omega$-saturated by Theorem 4.6.

\section{References}

[1] Baldwin, J. T., and D. W. Kueker, "Algebraically prime models," Annals of Mathematical Logic, vol. 20 (1981), pp. 289-330. Zbl 0494.03024. MR 83d:03042.

[2] Cawthorne, S., Forcing with Directed Families of Finite Structures, PhD thesis, University of Maryland, College Park, 1998. 
[3] Keisler, H. J., "Forcing and the omitting types theorem," pp. 96-133 in Studies in Model Theory, volume 8 of MAA Studies in Mathematics, Mathematical Association of America, Buffalo, 1973. MR 49:2340.

[4] Kueker, D. W., and M. C. Laskowski, "On generic structures," Notre Dame Journal of Formal Logic, vol. 33 (1992), pp. 175-83. Zbl 0768.03010. MR 93k:03032.

[5] Robinson, A., "Forcing in model theory," pp. 69-82, in Symposia Mathematica, volume 5, Academic Press, London, 1971. Zbl 0212.01802. MR 43:4651.

Department of Mathematics and Computing Sciences Marymount University 2807 North Glebe Road Arlington VA 22207

stephanie.cawthorne@marymount.edu

Department of Mathematics

University of Maryland

College Park MD 20742

dwk@math.umd.edu 